\title{
AUTHOR INDEX - VOL 44 - 2016
}

Abayaratne C.P., Wickramarathna A.V.U.A., Rodrigo D.D. \& Mannathunga K.S. - A low-cost partially automated polarimeter for investigating skylight polarisation 44: 95-103 (2016)

Abeyratne S.G., Perera N., Jayakody H., Samarakoon K. \& Bulathge R. - Zero-current-switching pulse charger for high capacity batteries in renewable energy applications 44: 301-312 (2016)

Afzal M.T. see Sajid N.A. et al. (2016)

Ahsan M. see Malook S.U. et al. (2016)

Ali Q. see Malook S.U. et al. (2016)

Amarasinghe A.D.U.S. see Jinasena M.A.M. et al. (2016)

Amarasinghe A.D.U.S. see Udana H.P.K. \& Amarasinghe A.D.U.S. (2016)

Amarasinghe B.M.W.P.K. see Jinasena M.A.M. et al. (2016)

Amaratunga D. \& Cabrera J. - High-dimensional data 44: 3-9 (2016)

Andersen R.J. see Ratnaweera P.B. et al. (2016)

Anthonys G. - The magnetisation of two ferromagnetic spheres placed randomly in a magnetic field 44: 23-32 (2016)

Asghar S. see Hussain T. \& Asghar S. (2016)

Asgher M. see Bilal M. \& Asgher M. (2016)

Aslam M. see Tahir M. et al. (2016)

Bamunusingha B.A.N.N., De Silva E.C.L. \& Gunasekera M.Y. - Performance of ion exchange resin as solid catalyst for the esterification of acetic acid with ethanol 44: 83-93 (2016)

Bandaranayake W.M.E.K., Wickramaarachchi W.A.R.T., Wickramasinghe H.A.M., Rajapakshe R.G.A.S. \& Dissanayake D.M.K.K. - Molecular detection and characterisation of bipartite begomoviruses associated with cucurbitaceous vegetables in Sri Lanka 44: 379-384 (2016)

Bilal M. \& Asgher M. - Biodegradation of agrowastes by lignocellulolytic activity of an oyster mushroom, Pleurotus sapidus 44: 399-407 (2016)

Bulathge R. see Abeyratne S.G. et al. (2016)

Cabrera J. see Amaratunga D. \& Cabrera J. (2016)

De Costa D.M. see Rienzie K.D.R.C. et al. (2016)

De Silva E.C.L. see Bamunusingha B.A.N.N. et al. (2016)

de Silva E.D. see Ratnaweera P.B. et al. (2016)

Dilrukshi I.A.D.N. \& Ranwala S.M.W. - Kirigala forest fragments and the identity as a dipterocarp plantation or Hora Kele of Ingiriya 44: 313-327 (2016)

Dissanayake P.B.R. see Dissanayake D.M.A.G.B. et al. (2016)

Dissanayake D.M.A.G.B., Kurukulasuriya L.C. \& Dissanayake P.B.R. - Evaluation of shear strength parameters of rail track ballast in Sri Lanka 44: 61-67 (2016)

Dissanayake D.M.K.K. see Bandaranayake W.M.E.K. et al. (2016)

Ekanayake E.W.M.A. see Menikarachchi M.A.S.K. et al. (2016)

Ekanayake M.P.B. see Perera P.H. et al. (2016)

Ekanayake M.P.B. see Ratnayake T.A. et al. (2016)

Fernando P. see Perera O.S. et al. (2016)

Fernando T.H.P.S., Senaviratne P., Siriwardane D. \& Madushani H.K.I. - White root disease of Murraya koenigii from Sri Lanka caused by Rigidoporus microporus 44: 347-348 (2016) 
Fernando W.S.K. see Perera P.H. et al. (2016)

Godaliyadda G.M.R.I. see Perera P.H. et al. (2016)

Godaliyadda G.M.R.I. see Ratnayake T.A. et al. (2016)

Goonasekara C.L. see Pushpakumara P.D. et al. (2016)

Gunasekera M.Y. see Bamunusingha B.A.N.N. et al. (2016)

Gunathilake T.R. see Vidhanaarachchi V.R.M. et al. (2016)

Gunatilake J. see Herath D. et al. (2016)

Hemachandra K.S. \& Perera M.C.D. - Assessment of the egg parasitoid guild of rice leaf folder, Cnaphalocrocis medinalis (Guenee): a search for biocontrol agents 44: 185-192 (2016)

Herath D., Pitawala A. \& Gunatilake J. - Heavy metals in road deposited sediments and road dusts of Colombo Capital, Sri Lanka 44: 193-202 (2016)

Herath H.M.S.P.B. see Perera P.H. et al. (2016)

Herath H.M.T., Rajapakse D., Wimalasena S. \& Weerasooriya M.K.B. - Zinc content and prediction of bio-availability of zinc in some locally grown rice (Oryza sativa L.) varieties in Sri Lanka 44: 291-299 (2016)

Hussain T. \& Asghar S. - Chi-square based hierarchical agglomerative clustering for web sessionization 44: $211-222$ (2016)

Hussain Z. see Tahir M. et al. (2016)

Jahan M.A.C.A. \& Ragel R.G. - Reproducing tables in scanned documents 44: 367-377 (2016)

Jayakody H. see Abeyratne S.G. et al. (2016)

Jayathilake Y.M.C.D. see Madhushani W.V.T. et al. (2016)

Jayawardana B.C. see Perera O.S. et al. (2016)

Jinasena M.A.M., Amarasinghe A.D.U.S., Amarasinghe B.M.W.P.K. \& Prashantha M.A.B. - Extraction and degradation of chlorophyll $a$ and $b$ from Alternanthera sessilis 44: 11-21 (2016)

Kapilan R. - Characterisation of purified protease from Bacillus subtilis BS166 44: 243-248 (2016)

Kapoor D.S. see Singh K.J. et al. (2016)

Karunaratne D.N. see Menikarachchi M.A.S.K. et al. (2016)

Karunaratne V. see Menikarachchi M.A.S.K. et al. (2016)

Katuwavila K.A.N.P. see Menikarachchi M.A.S.K. et al. (2016)

Kaushalya C.H.T.C. see Wickramarachchi P.A.S.R. et al. (2016)

Kohli A.K. see Ratan R. et al. (2016)

Kohli A.K. see Singh K.J. et al. (2016)

Kohli P.G. see Ratan R. et al. (2016)

Kulasooriya S.A. \& Magana-Arachchi D.N. - Nitrogen fixing cyanobacteria: their diversity, ecology and utilization with special reference to rice cultivation 44: 111-128 (2016)

Kulatunga D.D.S. see Sasabuchi S. \& Kulatunga D.D.S. (2016)

Kurukulasuriya L.C. see Dissanayake D.M.A.G.B. et al. (2016)

Liyanage G.Y. \& Manage P.M. - Isolation and characterisation of oil degrading bacteria from coastal waters and sediments from three locations in Sri Lanka 44: 351-358 (2016)

Liyanage R. see Perera O.S. et al. (2016) 
Lokupitiya E. - Country-specific emission factors for methane emission from enteric fermentation: a case study from a non-annex 1 country $\mathbf{4 4}$ : 137-144 (2016)

Madhushani W.V.T., Jayathilake Y.M.C.D., Perera K.S. \& Vidanapathirana K.P. - Effect of cation size of iodide salt in the electrolyte on the performance of dye sensitised solar cells 44: 77-81 (2016)

Madushani H.K.I. see Fernando T.H.P.S. et al. (2016)

Magana-Arachchi D.N. see Kulasooriya S.A. \& Magana-Arachchi D.N. (2016)

Malook S.U., Ali Q., Ahsan M., Shabaz M.K., Waseem M. \& Mumtaz A. - Combining ability analysis for evaluation of maize hybrids under drought stress 44: 223-230 (2016)

Manage P.M. see Liyanage G.Y. \& Manage P.M. (2016)

Mannathunga K.S. see Abayaratne C.P. et al. (2016)

Menikarachchi M.A.S.K., Katuwavila K.A.N.P., Ekanayake E.W.M.A., Thevanesam V., Karunaratne V. \& Karunaratne D.N. - Release behaviour of amoxicillin from chitosan coated liposomes derived from eggs 44: 167-173 (2016)

Mohotti K.M. see Senanayake P.D. et al. (2016)

Mohotti K.M. see Senanayake P.D. et al. (2016)

Mumtaz A. see Malook S.U. et al. (2016)

Muntakim A.H., Siddiquee M.S.A. \& Udagepola K.P. - Finite element simulation of the effect of loading rate on the stress-strain behaviour of Albany sand 44: 203-209 (2016)

Nettasinghe D.B.W. see Ratnayake T.A. et al. (2016)

Paranagama P.A. see Senanayake P.D. et al. (2016)

Paranagama P.A. see Senanayake P.D. et al. (2016)

Paranagama P.A. see Wickramarachchi P.A.S.R. et al. (2016)

Perera H.K.I. - PAGE - a simple method to detect the protective effects of medicinal plants against sugar induced protein damage 44: 105-107 (2016)

Perera K. see Rajapaksha K.W.G.D.H. \& Perera K. (2016)

Perera K.S. see Madhushani W.V.T. et al. (2016)

Perera M.C.D. see Hemachandra K.S. \& Perera M.C.D. (2016)

Perera N. see Abeyratne S.G. et al. (2016)

Perera O.S., Liyanage R., Weththasinghe P., Jayawardana B.C., Vidanarachchi J.K., Fernando P. \& Sivakanesan R. - Modulating effects of cowpea incorporated diets on serum lipids and serum antioxidant activity in Wistar rats 44: 69-76 (2016)

Perera P.H., Herath H.M.S.P.B., Fernando W.S.K., Ekanayake M.P.B., Godaliyadda G.M.R.I. \& Wijayakulasooriya J.V. - Online tracking and event clustering for vision systems 44: 385-397 (2016)

Pitawala A. see Herath D. et al. (2016)

Prabodanie R.A.R. - Optimal positioning of groundwater supply wells in residential areas with uncertain information 44: $233-242$ (2016)

Prashantha M.A.B. see Jinasena M.A.M. et al. (2016)

Premarathne P.H. see Pushpakumara P.D. et al. (2016)

Pushpakumara P.D., Premarathne P.H. \& Goonasekara C.L. - Characterisation of B cell epitopes of dengue virus NS1 protein using bioinformatics approach 44: 417-425 (2016)

Qadir M.A. see Sajid N.A. et al. (2016)

Ragel R.G. see Jahan M.A.C.A. \& Ragel R.G. (2016)

Rajapakse D. see Herath H.M.T. et al. (2016) 
Rajapaksha K.W.G.D.H. \& Perera K. - Wind speed analysis and energy calculation based on mixture distributions in Narakkalliya, Sri Lanka 44: 409-416 (2016)

Rajapakshe R.G.A.S. see Bandaranayake W.M.E.K. et al. (2016)

Ranwala S.M.W. see Dilrukshi I.A.D.N. \& Ranwala S.M.W. (2016)

Rasangika W.P.M. see Wickramarachchi P.A.S.R. et al. (2016)

Ratan R., Kohli P.G., Sharma S.K. \& Kohli A.K. - Un-supervised segmentation and quantisation of malignancy from breast MRI images 44: 437$442(2016)$

Ratnaweera P.B., de Silva E.D., Wijesundera R.L.C. \& Andersen R.J. - Antimicrobial constituents of Hypocrea virens, an endophyte of the mangrove-associate plant Premna serratifolia L. 44: 43-51 (2016)

Ratnayake S.P. - Bioconcentration modelling of alcohol ethoxylates by quantitative structure activity relationship approach: a first look 44: 443-450 (2016)

Ratnayake T.A., Nettasinghe D.B.W., Godaliyadda G.M.R.I., Ekanayake M.P.B. \& Wijayakulasooriya J.V. - An information rich subspace separation for non-stationary signal classification 44: 257-271 (2016)

Rienzie K.D.R.C., Wickramaarachchi W.A.R.T., De Costa D.M. \& Wijesooriya W.M.G.U. - Molecular detection and characterisation of begomovirus causing bean yellowing disease in Sri Lanka 44: 249-255 (2016)

Rodrigo D.D. see Abayaratne C.P. et al. (2016)

Sajid N.A., Afzal M.T. \& Qadir M.A. - Multi-label classification of computer science documents using fuzzy logic 44: 155-165 (2016)

Samarakoon K. see Abeyratne S.G. et al. (2016)

Samaratunge I.N. see Wickramarachchi P.A.S.R. et al. (2016)

Sasabuchi S. \& Kulatunga D.D.S. - On the powers of tests for homogeneity of regression coefficient vectors under synchronised order restrictions 44: 53-60 (2016)

Senanayake P.D., Mohotti K.M. \& Paranagama P.A. - Identification and substrate utilisation of fungi associated with low country live wood termite, Glyptotermes dilatatus Bugnion \& Popoff and the host plant, Camellia sinensis L.O. Kuntze 44: 175-184 (2016)

Senanayake P.D., Mohotti K.M. \& Paranagama P.A. - Volatile constituents of tea stems (Camellia sinensis L.O. Kuntze) as semiochemicals to attract low country live wood termite, (Glyptotermes dilatatus Bugnion \& Popoff) in Sri Lanka 44: 33-42 (2016)

Senaviratne P. see Fernando T.H.P.S. et al. (2016)

Shabaz M.K. see Malook S.U. et al. (2016)

Sharma A. see Singh K.J. et al. (2016)

Sharma S.K. see Ratan R. et al. (2016)

Siddiquee M.S.A. see Muntakim A.H. et al. (2016)

Singh K.J., Kapoor D.S., Sharma A. \& Kohli A.K. - Multi-level threshold based edge detector using logical operations 44: 145-154 (2016)

Siriwardane D. see Fernando T.H.P.S. et al. (2016)

Sivakanesan R. see Perera O.S. et al. (2016)

Somathilake L.W. \& Wedagedera J.R. - Investigation of branching structure formation by solutions of a mathematical model of pattern formation in coral reefs 44: 427-436 (2016)

Suranjith W.C. see Vidhanaarachchi V.R.M. et al. (2016)

Tahir M., Aslam M. \& Hussain Z. - Estimation of parameters of the 3-component mixture of Pareto distributions using type-I right censoring under Bayesian paradigm 44: 329-345 (2016)

Thevanesam V. see Menikarachchi M.A.S.K. et al. (2016)

Udagepola K.P. see Muntakim A.H. et al. (2016) 
Udana H.P.K. \& Amarasinghe A.D.U.S. - Evaluation of single bed and multi bed dried copra on the quality of extracted coconut oil 44: $359-365$ (2016)

Vidanapathirana K.P. see Madhushani W.V.T. et al. (2016)

Vidanarachchi J.K. see Perera O.S. et al. (2016)

Vidhanaarachchi V.R.M., Suranjith W.C. \&. Gunathilake T.R. - Effect of genotype, embryo maturity and culture medium on in vitro embryo germination of Sri Lankan coconut (Cocos nucifera L.) varieties 44: 273-278 (2016)

Waseem M. see Malook S.U. et al. (2016)

Wedagedera J.R. see Somathilake L.W. \& Wedagedera J.R. (2016)

Weerasooriya M.K.B. see Herath H.M.T. et al. (2016)

Weththasinghe P. see Perera O.S. et al. (2016)

Wickramaarachchi W.A.R.T. see Bandaranayake W.M.E.K. et al. (2016)

Wickramaarachchi W.A.R.T. see Rienzie K.D.R.C. et al. (2016)

Wickramarachchi P.A.S.R., Samaratunge I.N., Kaushalya C.H.T.C., Rasangika W.P.M. \& Paranagama P.A. - Activity of chitosan films enriched with the essential oil of Alpinia malaccensis rhizome against S. aureus, E. coli and C. musae 44: 129-135 (2016)

Wickramarathna A.V.U.A. see Abayaratne C.P. et al. (2016)

Wickramasinghe H.A.M. see Bandaranayake W.M.E.K. et al. (2016)

Wijayakulasooriya J.V. see Perera P.H. et al. (2016)

Wijayakulasooriya J.V. see Ratnayake T.A. et al. (2016)

Wijesooriya W.M.G.U. see Rienzie K.D.R.C. et al. (2016)

Wijesundera R.L.C. see Ratnaweera P.B. et al. (2016)

Wijeyaratne W.M.D.N. - Application of pollution indices to quantify the pollution status of shallow sediments of the Bolgoda Lake, Sri Lanka 44: 279-289 (2016)

Wimalasena S. see Herath H.M.T. et al. (2016) 